\title{
Reconstructing Macroeconomics, A Perspective from Statistical Physics and Combinatorial Stochastic Processes: Book Review
}

\author{
Narela Bajram \\ Faculty of Business and Administration, International University of Sarajevo, \\ Hrasnicka Cesta 15, Ilidza, 71200 Sarajevo BiH, \\ narela7@yahoo.com.
}

\begin{abstract}
Professors Aoki and Yoshikawa adopted a variety of concepts from statistical physics and combinatorial stochastic processes to various problems in economics (such as labor markets, real growth, consumption, unemployment, financial market, productivity difference, etc.). In order to analyze these phenomena, they depart from the standard methods of model construction and analysis in mainstream economics and use methods that generally fall into two broad categories. One deals with stochastic dynamics, and the other with the formation of clusters and random combinatorial analysis.

The authors build their new macroeconomics on the observation that the huge number of heterogeneous agents act stochastically different based on individual insights, tastes, goals, etc. In this approach the properties of economic systems are described by macroscopic dynamical equations of motion that are nonlinear partial differential equations, such as backward and forward Kolmogorov equations (known as a Chapman-Kolmogorov equation and Fokker-Planck equations).
\end{abstract}

Keywords—Equilibrium and non-equilibrium, jump Markov process, master equation and uncertainty trap. 


\section{BOOK REVIEW}

Title: Reconstructing Macroeconomics, A Perspective from Statistical Physics and Combinatorial Stochastic Processes

\section{Authors:}

1. Masanao Aoki, Professor Emeritus in the Department of Economics at the University of California, Los Angeles

2. Hiroshi Yoshikawa, Professor of Economics at the University of Tokyo.

Publisher: Cambridge University Press 2007

\section{INTRODUCTION}

The authors of this book adopted a variety of concepts from statistical physics and combinatorial stochastic processes to various problems in economics (such as labor markets, real growth, consumption, unemployment, financial market, productivity difference and etc.). This book is concerned with understanding the properties of economic systems described by macroscopic dynamical equations of motion that are nonlinear partial differential equations, such as backward and forward Kolmogorov equations (known as a Chapman-Kolmogorov equation and Fokker-Planck equations).

As expressed by Stanley et al (1996), "Statistical physicists have determined that physical systems which consist of a large number of interacting particles obey universal laws that are independent of the macroscopic details". The new theory in this book is based on the following postulates: i) economies have a large number of stochastically interacting agents and the notion that an economy can be described in terms of a single representative agent is at best a very rough approximation. ii) statistical mechanics suggests that uncertainty in not just important at the level of individual decision making, but also at the level of collective behavior, and in circumstances where agents may not be fully rational (in this state traditional policies are ineffective). iii) prices are inflexible and this is caused by the slow dynamics of macro system iv) equilibrium is analyzed as probability distribution nor as a point. Real variables such as the growth rate of GDP or consumption obey exponential distribution, while asset prices and returns obey power-law distribution.

But before introducing the Aoki's and Yoshikawa approach in reconstructing the macroeconomics we should first explain what we mean by the terms equilibrium and non-equilibrium in the context of macroscopic systems.

If the properties of a thermodynamic system are independent of time it is said to be in a steady state. In this stage noting is happening. Equilibrium systems generally are closed systems, or systems in contact in which the pressure or temperature are constant. Thus, a system that is neither simply isolated, nor in contact with a constant temperature will be non-equilibrium. A system that is in the transient before reaching equilibrium is also in non-equilibrium. The non-equilibrium state may also be maintained by external fields/forces and features that may be associated with non-equilibrium are dynamics.

A more subtle, but equally important difference between equilibrium and non equilibrium systems is the role of fluctuations. In equilibrium they are Gaussian, large deviation from equilibrium are very rate and fluctuations do not give rise to macroscopic currents and they are in arrow of time. Bergersen (2009) point out that if a film is made of a series of fluctuating events and the film is afterward run backwards, and an observer will be unable to tell the difference. In contrast, fluctuations in non-equilibrium systems frequently have a rectifying effect, providing a sense of direction. He also states that in many ways the lack of concern with dynamics makes the general equilibrium theory of economics like meteorology without the weather.

Modern economists often apply equilibrium concepts in which supply meets demand and markets are cleared, perhaps guided by Adam Smith's invisible hand. Moreover, in the $20^{\text {th }}$ century the economics Nobel Prize was given mostly to neoclassical economist applying the general equilibrium theory.

The authors of this book it seems that are more concerned with what actually happened then with what theory and ideology predicts and they argue that better analogies can be found in modern non-equilibrium statistical mechanics. Due to the persistent exchanges of information between the system and its environment each particle (agent) is not free and it is always feeling the influences from the interactions with other particles (agents) in the system and the environment. Also they emphasize the importance of phase transitions because they play an equally important role in the formulation of structures in nonequilibrium systems as in the equilibrium systems. Consequently, temperature (aggregate demand in the book) is no longer uniform but may be with a distribution. In physics it is frequently observed that the relaxation dynamics of nonequilibrium systems can be governed by an exponential law or power-law. When the number of micro growth event within a period is small, and exponential distribution can emerge. But when the number of micro growth events within a period is large, then power-law distribution can emerge. Based on this, Aoki and Yoshikawa point that rarely happens that our salaries and wages are doubled within a relatively short period of time, say a year. In contrast, very often the price of a stock can double in a year. This difference is formally reflected in two different probability distribution. One is exponential distribution for the growth rate of real variable such as GDP or consumption, while the other is power-law distribution for asset prices and returns.

In order to analyze these phenomena, they depart from the standard methods of model construction and analysis in mainstream economics and use methods that generally fall into two broad categories. One deals with stochastic dynamics, and the other with the formation of clusters and random combinatorial analysis.

Stochastic dynamics examines behavior of the model over time and extract dynamic properties of stochastic models. The key method is to solve evolution equations for probability 
distribution such as the Chapman-Kolmogorov equation or master equation, and the Fokker- Plank equation. The master equation gives us stationary or equilibrium behavior of the model and fluctuations around equilibrium are obtained by solving the associated Fokker-Plank equation.

Random cluster formation deals with combinatorial aspect of agents forming clusters. This has to do with the sizes of configurations or state spaces. Using these methods, we can examine the size distribution of frequency spectrum.

The methods used to analyze the macroeconomics and financial markets share one or more of the following three features:

1. A finite but large number of micro units or agents interact

2. Different "types" of agents are present

3. New and unknown types of agents, products may appear, that is , the number may not be fixed nor known in advanced.

\section{Agent Representative Model (RAMSAy AND DiAmond} MODEL) VS AOKI AND YOSHIKAWA'S MODEL

In the lecture "What is Life? held by Schrodinger (1944), a physicist and one of the founders of the quantum mechanics, noted that macroeconomic regulations emerge not from microeconomic behavior of the representative agent but from stochastic interaction of a large number of agents. The standard model described in the macroeconomic textbooks first considers microeconomic behavior of the representative model, and then translate essentially microeconomic behavior equations into macroeconomic equations simply by adding stochastic terms. Additionally, focuses on means and variances of the variables of interest and such a example is the Real Business Cycle model. In physics, the law of motion stands only for micro units and does not really help our understanding of macro systems. Therefore, we need different approach and in this book the author note that it is often necessary to look at sample paths and probability distribution, not just moments of stochastic variables to understand the behavior of the macroeconomic system.

In 1928 a sophisticated model of a society's optimal saving was published by the British mathematician and economist Frank Ramsey. Ramsey contribution was mathematically demanding and did not experience much response until 1956 when his contribution was taken up seriously by Samuelson and Solow. In the mid 1960 the model was fashioned with Solow's simpler growth model and become a cornerstone in the neoclassical growth theory. But in the some period another model appears, known as a Diamond's model.

In the Diamond model there is an infinite number of agents (in every new period a new generation enters) and these have a finite time horizon. In contrast, in the Ramsey model there are finite numbers of agents with an infinite time horizon. Thus, the Ramsey model is a representative agent model, whereas the Diamond model has heterogeneous agents interacting in every period.
Moreover in the Ramsey model there are no stochastic elements. It is assumed that households understand precisely how the economy works and can predict the future path of interest rates and wages. Because of the rational expectations the uncertainty is assumed absent so the Ramsey model always generates unique equilibrium paths. On the other hand, in the Diamond model under certain circumstances multiple equilibrium paths could arise.

Thus, we can not explain the behavior of consumers by taking in consideration only the micro behavior of one consumer, as shown in the Ramsey model (Figure 1). The economy is a complex system and is helpless to describe the micro behavior of all the consumers. In such a case, we cannot deduce the behavior of the macro system from that of the micro units.

In the book is shown that as a result of the Ramsey-type optimization subject to shock to economic utility function, some households increase while others lower their consumption. (see Figure 1).

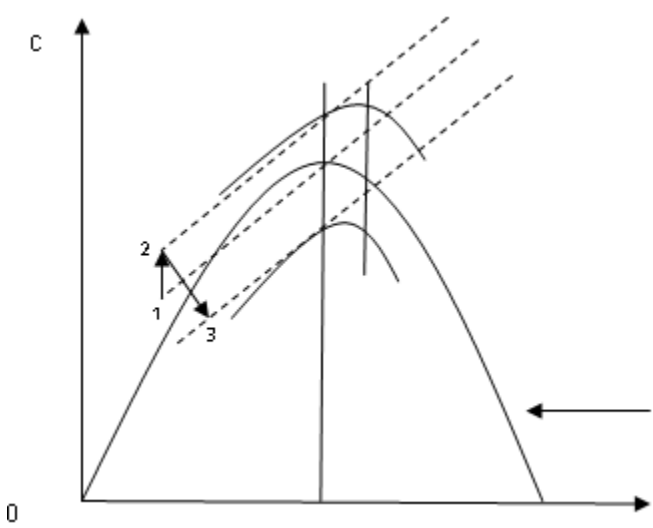

Figure 1. The Behavior of a Ramsay Consumer in Stochastic Environment

Note: The explanation of this phase diagram can be found in the advanced textbook of macroeconomics such as Blanchard and Fischer (1989).

Therefore it is useful to classify the types of household, and to assume that household types change in stochastic way. But the classification cannot be done by Diamond model who introduce two types of agents; the employed and the unemployed, because in each group, there is only the representative agent. There are no microeconomic fluctuations within each group.

The model in this book and the Diamond model are obviously quite similar where the agents faces uncertainty and their behavior is stochastic but the difference arises from the existence of microeconomic fluctuations in the model presented in the book. As we munched above in the standard Diamond model the problem of equilibrium selection arises, because the equilibrium is explained as a point not as a distribution. In contract, in the approach used in the book this problem of selection does not arise. The macroequilibirum is observed as a probability distribution and describe by a partial differential equation (Chapman-Kolmogorov equation) instead of an ordinary differential equation. 


\section{EQUILIBRIUM AS A DISTRIBUTION}

Thus the statistical physics applies not only to physics and chemistry but also to economic (Schrodinger's observation). This is due the fact that the law for macro systems is also statistical. Further in the text is explained how an approach on statistical physics can be applied to macroeconomics. At the beginning they resort to a stochastic approach and classify the types or states of microeconomic agents, and then analyze stochastic transitions of such types or states to draw important implications for the economy as a whole. In the book a continuous-time Markov chain also known as a jump Markov processed is used. The models have countable states and they are specified by sets of transition rates which can be nonlinear functions of state variables, called state-dependent transition rates.

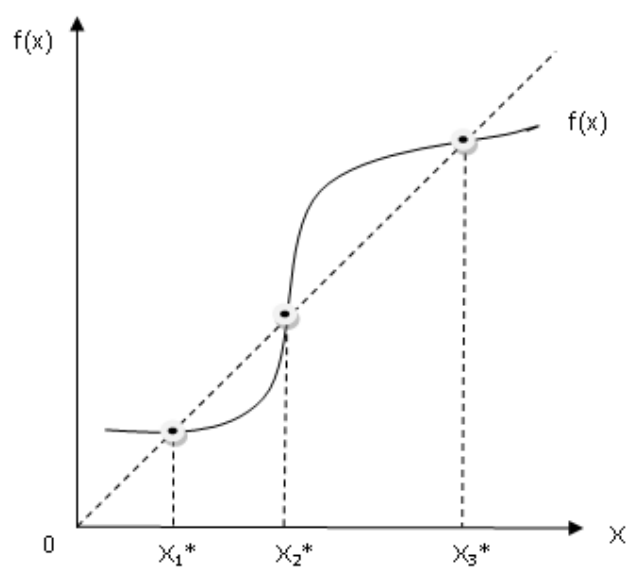

Figure 1.5. Multiple Equilibrium

By using the state-dependent transition rates multiple equilibrium can be produced. (see Figure 2) and the economy then stochastically fluctuates between $\mathrm{x} 1$ and $\mathrm{x} 3$ which are known as stable equilibriums. But as we already munched above this model provides a possible explanation of fluctuations of the economy, and the problem of selection of equilibrium in such a situation does not arise. Also what is important to be emphasized is that often a certain macro fluctuations or pattern is not similar to those of micro units.

In Chapter 3 is proposed a new concept of equilibrium. In the standard analysis, because the macroeconomic equilibrium is explained by a point in some space or set, its evaluation is described by an ordinary differential equation. In this chapter the equilibrium is analyzed as a probability distribution, not a simple point. This approach requires a partial differential equation to describe evolution of a macroeconomic equilibrium over time (this is the Chapman-Kolmogorov equitation or in the book known as a master equation).

Aoki in his book argues that:

Instead of analyzing optimizing behavior of individual agets we must use proper statistical methods suitable for the study of the macro system consisting of a large number of fluctuating micro units. We maintain that the so-called micro foundation for macroeconomics are not true micro foundations, but simply misguide.
Thus, the proper microeconomic foundations for macroeconomics are given by specify transition rates for the jump Markov process. These are so called Boltzmann-Gibbs type transition rates. The special binary choice models are actually consistent with stochastic dynamic optimization commonly used in economics.

The model presented in this chapter in fundamentally different from the New Keynesian Economist who focus in explaining the price/wage rigidity and Neoclassical Economist (based on Ramsey consumer). Aoki and Yoshikawa concentrate on the effective demand but they also take in consideration the saturation effect. Moreover, in the neoclassical theory because of markets efficiency, the productivity differential is a puzzle, while in the Aoki's models it is considered as a necessity in the macroeconomy. We cannot suppose that all the economic agents and production factors move instantaneously to the sector with the highest productivity. Differences in productivity remains in the economy at all time. Therefore the behavior is explained with transition rates. Consequently, at each moment we have a range of productivity levels. Because aggregate demand affects the transition rates of production factors across sectors/firms with different productivities we can conclude also that aggregate demand affect aggregate output. It is also important to be recognized that productivity depends not only on technology or consumers preferences but also on changes of production factors. And the aggregate demand in Aoki and Yoshikawa model corresponds to temperature in physics.

He shows that in equilibrium, the distribution of production factors across sectors with different productivity is of the Bolzman-Gibbs type:

$$
\frac{n i}{N}=\frac{e^{\frac{-N c i}{D}}}{\sum_{i=0}^{n} e^{\frac{N c i}{D}}} \quad \mathrm{i}=1, \ldots . ., \mathrm{s}
$$

Where $\mathrm{n}$ is the amount of production factor used in sector $\mathrm{i}, \mathrm{N}$ is its total endowment, $c$ is the productivity coefficient in sector $i$, and $\mathrm{D}$ is the aggregate demand. The Bolzman-Gibbs distribution means that when the aggregate demand is high, production factors are mobilized to higher productivity. A similar point is made by Okun (1973). An important result of econophysics is that the Botzmann-Gibbs distribution is not affected by the specific characteristics of the agents involved in the economic model (Dragolescu and Yakokenko, 2000).

\section{UNCERTAINTY TRAP AND THE EFFECTIVENESS OF THE POLICY}

In Chapter 4 they focus on particular factor-uncertainty. When the economy is trapped into bad equilibrium the uncertainty rises. In the classical textbook it is assumed that the uncertainty is insignificant and the economy fluctuates around the unique/natural equilibrium and policies are effective. In contrast, standard macroeconomic polices necessarily become ineffective. Indeed, the "uncertainty trap" is what distinguishes "depression" from normal "recession". These analyses are applied to the long stagnation of the Japanese economy during the 1990s. We can 
notice that even the interest rate approaches 0 the economy is not recovering (the polices are ineffective). The model used in this chapter is the some presented in Chapter 3 (binary choice model).

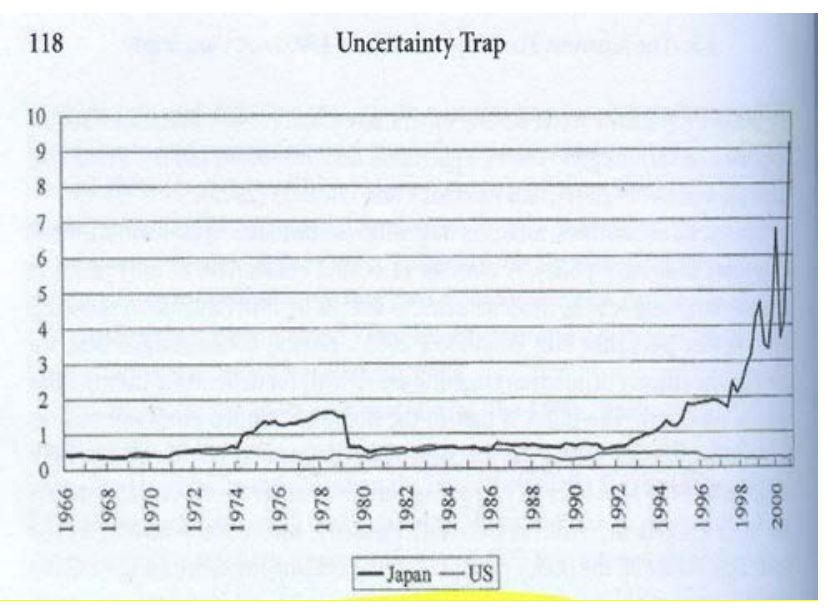

Figure 3. CV of Growth Rate of GDP for Japan and United States.

The degree of uncertainty $(\beta)$ is measured by using the country's GDP growth rates and calculates the variation. The Figure 3 shows the coefficient of variation (CV=Standard Deviation/ Mean) of the quarterly GDP growth for 5 years in Japan and US. We can notice that in Japan the degree of uncertainty has been risen. In this case we must depart from the standard representative agent assumptions, and seriously consider the stochastic approach with a large number of economic agents.

\section{SLOW DYNAMICS, INFLEXIBLE PRICES AND SATURATION OF DEMAND IN GROWTH THEORIES}

Chapter 5 presents the slow dynamics of macro system, as a example are taken inflexible prices. The standard approach shows that the rational agents respond quickly to any change in the economic environment. Many economists take inflexible prices as an irrational behavior of the agents. But the price inflexibility in fact is generic property of any complex economic system.

Aoki and Yoshikawa the slow dynamics consider also as a necessity in the macroeconomics.

They explain this phenomena using tree structure in which levels are basic clusters/sectors of agents. They focus on the fact that sectors are not symmetric or that the distance difference across sectors. The traditional measure for strength of relationship between two variables is the correlation, but is not transitive. To avoid this intransitive in this chapter the authors use the ultrametric or tree distance as a measure for distance between sectors or cluster of agents. The ultra-metric measure used in the literature of mathematic is introduced into the jump Markov process. The transition rate from one state to another state is small when the ultra-metric distance is large and vice versa. They demonstrate that such a hierarchy structure of clusters/states necessarily produces slow adjustment (such as inflexibly prices). Before we proceed, it is interesting to compare the behavior of quantities and prices.
Analyzing the adjustment of quantities and prices it is pointed out by Clower (1965) and Leijonhufvud(1968) that the quantity adjustment might be actually much faster than price adjustment. This trend is also shown in the book by analyzing the variance of distribution in the Japanese manufacturing industry and transport machinery industry.

Although studies of macro economy with many agents are not new, dynamic behavior in disequilibrium has not been satisfactory analyzed. And in the literature this analyzes are known as "Non-Walrasian" or disequilibrium analyzes such as Benassy (1975) and Malinvaud(1977). However, in this models dynamic is not considered because their purpose was just to show the existence of quantity-constrained equilibrium. The model presented in this book as the author's state share the spirit of "Non-Walrasian" approach, but with dynamics. They considered very simple quantity adjustment model with a large number of sectors or agents and the sectors have different productivities. In addition, resources are stochastically allocated to sectors depending on excess demands or supplies of the sector. This model shows that the total output of such an economy fluctuations, and that the average level of aggregate production (GDP), depends of demand. When the excess demand of all sectors is equal to 0 , then the output also stays unchanged. We can notice this stochastic model clear relationship between demand and output emerges. If we consider two sectors model where one is more productive then the other sector, then higher demand for goods and services for higher productive sectors will result higher expected value of output/GDP. In contract, if the demand is higher for less productive sectors then expected value for employment will be higher. But the key factor for economic growth by Aoki and Yoshikawa is the emergence of new sectors. This model demonstrated that cycles, in fact, are endogenously generated.

In the standard literature of growth, the fundamental factor restraining economic growth is diminishing returns to capital in production or technology. In this book saturation of demand is shown that is another important factor restraining growth. The demand for some product in the classical economics it is shown that grows exponentially. But in reality the demand grows according to the logistic curve. (see figure 4).

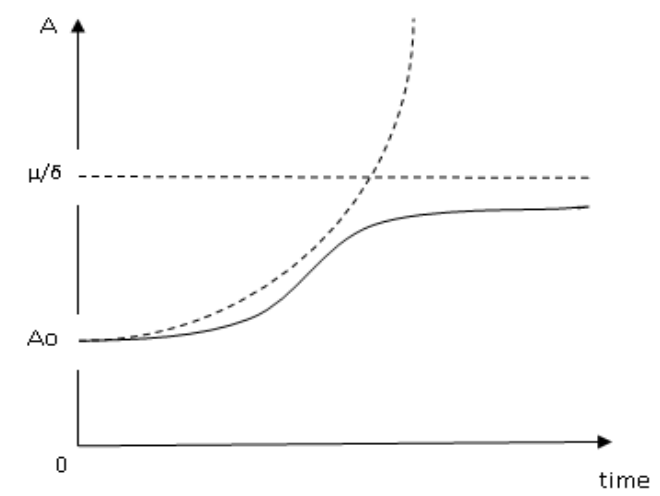

--- Logistic Growths ------ Exponential Growth Figure 4. The Logistic Curve 


\section{ANALYZING FinANCIAL MARKETS}

As we can notice the focus in this book is more on the real economy, but at the end Aoki and Yoshikawa show that this approach can be equally usefully also for analyzing the financial market.

They identify investors with the strategies or the rules they employ, and say that investors of the same type form a group or cluster. The clusters change as agents enter or exit. They conclude that when the number of participants is $n \geq 100$ then we can group them in two large clusters. The fluctuation of the price can be greater or lower depending on the clusters agents who dominate the market.

\section{SUMMARY}

In sum, by focusing on the most recent developments in the theory of nonlinear dynamics and their mutual relationship to stochastic dynamics as well as its applications in economics this book sets new standards in macroeconomics. I hope that this book will help us in better understanding of the real world around us and will lead to the development of new ideas and computational and theoretical approaches.

\section{REFERENCE}

Benassy, J.P. (1975),"Neo-Keynesian Disequilibrium Theory in a Monetary Economy”, Review of Economic Studies, Vol.42, 503-523.

Bergersen B. (2009), "Statistical Mechanics of Risk, Utility and Equilibrium; Analogies between Economics and Statistical Physics", University of British Columbia, Canada.

Clower R. (1965), “ The Keynesian Counterrevolution: A Theoretical Appraisal”, in F. Hahn and F. Brechling (eds), The theory of Interest rates, London: Macmillan.

Diamond P.(1982) "Aggregate Demand Management in Search Equilibrium”, Journal of Political Economy, Vol.90, 881-894.

Dragulescu A. and V. M. Yakovenko,(2000) "Statistical mechanics of money,” European Physical Journal B, vol. 17, no. 4, pp. 723- 729.

Leijonhufvud A. (1968) “ Keynesian Econmics and Economies of Keynes”, Oxford: Oxford University Press.

Malinvuad, E. (1977), “Theory of Unemployment Reconsidered, Basil Blackwell, Oxford.

Mallick K. (2009), "Some recent developments in non-equilibrium statistical physics”, Journal of Physics, Vol. 73, No.3, pp.417-451.

Okun, A.M.(1973), "Upward Mobility in a High-Pressure Economy,” Brooking Papers on Economic Activity, Vol.1, 207-261.

Ramsey, F. (1928), “ A Mathematical Theory of Saving”, Economic Journal, December, Vol. 38, 543-559.

Ruelle D. (2008), "What physical quantities make sense in nonequilibrium statistical mexhanics?”, in Boltzmann's Legacy edited by G. Gallavotti, W.L. Reiter, and J. Yngvason, European Mathematical Society, Zürich.

Schrodinger E. (1944),"What is Life?, Cambrigde: Cambrigde University Press.

Solomon S, and Richmond P.(2001), " Power Laws of Wealth, Market Order Volumes and Market Returns”, Physical A:Statistical Mechanics and its Applications, Vol. 229(1-2), 188-197.
Solow R.M. (1956) “ A contribution to the Theory of Economic Growth”, Quarterly Journal of Economics, Vol. 70, 65-94.

Stanley M.H.R., L.A.N. Amaral, S.V. Buldyrev, S. Havlin, H. Leschhorn, P.Maass, M.A. Salinger, and H.E. Stanley (1996)"Scaling Behavior in the Growth of Companies,” Nature, Vol. 379, 804-806.

Stanley, M.A. , Beck, J. G. \& Glassco, J.D. (1996), “ Tretment of generalized anxiety disorder in older adults: a preliminary comparison of cognitive-behavioral and supportive approaches”, Behavior Therapy, 27, 565-581.

Taylor, J.B. (1989), "Differences in Economic Fluctuations in Japan and the US: The Role of Nominal Rigidities," Journal of Japanese and International Economies, Vol. 3. 127-144.

Yoshioka D. (2007),” Statistical Physics: An Introduction”, Springer, New York. 